\title{
Zur Strafbarkeit von Blockaden in der jüngsten Rechtsprechung
}

Die Pershing-Raketen werden aufgestellt. Protest und Widerstand dagegen verliefen recht friedlich. Die Gründe dafür sind sicher vielfältig. Unter anderem dürfte das Verhalten der Polizei mitgewirkt haben. Im Zusammenhang der Strategie der Polizei sollen hier einige Gerichtsurteile vorgestellt werden. Sie betreffen Fragen der Nötigung durch Demonstranten vorwiegend der Friedensbewegung ${ }^{1}$. Die Sachverhalte sind allemal ähnlich: Bürger stehen oder sitzen auf der Straße, auf Zufahrtswegen zu militärischen Anlagen o. ä. und demonstrieren durch die Verkehrsbehinderung, durch Spruchbänder, Handzettel usw. gegen die militärische Aufrüstung. - Diskutiert wird auch das neue Urteil des Bundesgerichtshofes zur Auseinandersetzung um die Frankfurter Flughafenerweiterung (Fall Alexander Schubart), welches Fragen der Nötigung von Verfassungsorganen und der Täterhaftung von »Hintermännern « des Landfriedensbruchs betrifft.

\section{Polizeiliche Konfliktsteuerung und Definitionsmacht}

Angesichts der Demonstrationen der Friedensbewegung vermied es die Polizei oft nicht immer -, gewaltsam gegen die von den Demonstranten gebildeten Verkehrsblockaden vorzugehen. Sie leitete den öffentlichen Verkehr oft um. Das wirkt sich, von der Polizei vielleicht nicht beabsichtigt, auch in den einschlägigen Strafurteilen aus. Verurteilungen wegen Landfriedensbruchs und Widerstand, die zu erwarten gewesen wären, wenn die Polizei gegen die Blockaden schärfer vorgegangen wäre, sind kaum bekannt geworden. Wo der Verkehr umgeleitet wurde, ist nach Ansicht der Gerichte der Nötigungserfolg der Blockade nicht eingetreten ${ }^{2}$. In Betracht kommt dann nur versuchte Nötigung, die milder bestraft werden kann. Zudem ist der Nachweis des Nötigungsvorsatzes praktisch schwieriger, wenn das Delikt nicht vollendet wurde. Es fehlt dann die Grundlage für die in der Praxis verbreitete Annahme, was jemand bewirkt habe, das habe er im allgemeinen auch gewußt und gewollt. Außerdem haben, wenn es umständlicher und unsicherer wird, den Vorsatz nachzuweisen, die Richter größere Spielräume. Einem Richter, der die prinzipielle Rechtmäßigkeit von Demonstrationen anerkennt, fällt es dann leichter anzunehmen, der Vorsatz sei nicht mit der erforderlichen Sicherheit festzustellen. Der Richter

I Es handelt sich nur um eine vorläufige Bilanz. Besprochen werden einige Entscheidungen, die bis März 1984 mehr oder weniger zufällig zugänglich waren.

2 AG Stuttgart-B $33 \mathrm{Cs}_{2424} / 8_{3}$ - in KJ I 983 , S. 430 (433). (Das Urteil des AG Stuttgart vom 3. 8 . 1983 $\mathrm{B} 33 \mathrm{Cs}_{2423} / 83$ betrifft denselben Sachverhalt und lautet ebenso); AG Erlangen v. I 5 . $11.83-3$ DS 342 Js $35633 / 83$ - in StrVert 1984,28 f.; OLG Köln v. 28. 4. 83- $3 S_{1} 128 / 83$ in NJW $1983-2206 \mathrm{f}$. = StrVert 1983,373 f.; ähnlich LG Stuttgart v. 29.9.83-7 Qs $110 / 83$ - in StrVert 1984, 28. 
muß das nicht annehmen; die Beweiswürdigung ist frei. Verneint er den Vorsatznachweis, so setzt er die von der Polizei betriebene Konfliktvermeidung fort. Die Möglichkeit dazu gab zunächst der zurückhaltende Umgang der Polizei mit den Blockaden. Die Strategie der Polizei entschied also nicht nur über die unmittelbaren Folgen ihres Verhaltens (z. B. Beseitigen oder Zulassen einer Störung), sondern - mit oder ohne Willen der Polizei - auch über Entstehen und Ausmaß dessen, was als kriminell bestraft wird.

Die polizeiliche Strategie muß nicht immer darauf hinauslaufen, daß Kriminalisierung verringert wird. Bei der Blockade eines Munitionslagers hatte der Lagerkommandeur den Verkehr mit dem Lager zu einem nicht blockierten Tor verlegt. Deshalb wurde, wie das AG Erlangen ${ }^{3}$ feststellte, niemand in strafbarer Weise genötigt. Allerdings hatte während der Blockade der Kommandeur "auf Anraten der deutschen Polizei« einen Fahrer beauftragt zu erkunden, ob das Tor, vor welchem die Demonstranten saßen, wirklich noch blockiert werde. Der Fahrer fuhr zweimal bis zu den Demonstranten, wurde nicht durchgelassen und kehrte jeweils um. Das Amtsgericht verneint vollendete Nötigung, weil die Fahrt vermutlich nur Testzwekken gedient habe. Dann erwägt es weiter, das Geschehen lasse wauch den Schluß zu, daß der Zeuge (der Fahrer) sich nötigen lassen wollte, um eine Erfüllung des Tatbestandes der Nötigung herbeizuführen, da sonst aufgrund der Nebenstrecke (Umleitung) keine weiteren Fahrzeuglenker vorhanden gewesen wären, die durch die Blockade hätten genötigt werden können. Es handelt sich somit um eine provozierte Nötigung.« Das AG versagte der möglichen Provokation die Wirkung: "volenti non fit iniuria«. Hätte es die mögliche Provokation nicht durchschaut, wäre dieser Ausweg versperrt gewesen 4 .

Angesichts der Aktionen der Friedensbewegung verhielt sich die Polizei nicht nur reaktiv. Sie beschränkte sich nicht darauf, entstandene konkrete Gefahren abzuwehren und Straftaten aufzuklären, nachdem sie unabhängig von ihr begangen wurden. Sie verhandelte mit Vertretern der Friedensbewegung und handelte - dem Vernehmen nach - aus, sie werde sich zurückhalten, wenn die Demonstranten sich auf bestimmte Blockaden beschränken würden. Das Blockieren ist nach herrschender Rechtsprechung kriminelle Nötigung. Die Polizei nahm es in großem Umfang hin und leitete den Verkehr um. Sie beeinflußte das Geschehen und hatte die Macht, es in den Bereich der Kriminalität laufen zu lassen. Wenn man also die soziale und politische Bedeutung der zu den jüngsten Demonstrationen ergangenen Strafurteile verstehen will, ist es wichtig, die Strategie der Polizei zu berücksichtigen. Aus dieser Strategie u. a. sind die Urteile hervorgegangen.

I98I veröffentlichte die Zeitschrift CILIP eine Untersuchung der Konflikte um die damaligen Hausbesetzungen'. Danach setzte sich in der Polizei in den 7 oer Jahren eine neue Einsatzkonzeption durch. Nicht mehr die schematische Durchsetzung von Normen und Befehlen bestimmt ihr Handeln, sondern politische Kosten/NutzenErwägungen. Der Einsatz physischer Gewalt kann delegitimierend wirken. Wenn die harte und spektakuläre staatliche Machtentfaltung von der Öffentlichkeit mißbilligt wird, kann sie die Anerkennung der Polizei und der Regierung schwächen.

\footnotetext{
3 S. o. Fn. 2.

4 Eine Provokation durch die Polizei wäre nichts Neues. Die Provokation von Straftaten ist eine verbreitete und umstrittene Polizeipraxis. Sie hat eine lange Geschichte. Daß sie gegenwärtig u. a. im Bereich der Drogendelikte und des Waffenhandels stattfindet, ist bekannt; vgl. Dencker, Festschrift für Dünnebier 1982, S. 447 ff.; Lüderssen, Festschrift für Peters, 1974, S. 349 ff.; BGH NJW 198 1, 1626; CILIP (jetzt: Bürgerrechte u. Polizei. Hg. v. Narr, Funk, Busch, Kauß, Kunze, v. Zabern, Werkentin), H. I1, I98z, S. $57 \mathrm{ff}$.

s CILIP (s. o. Fn. 4), H. $9 /$ ro, 198 r, S. 83 ff., 148 ff.
} 
Deshalb berücksichtigt die Polizei neben der Legalität die politische Legitimität ihrer Einsätze. Ihr Handeln geht flexibel auf die Besonderheiten der jeweiligen politischen Situation ein. Es folgt nicht mehr schematischen Tatbeständen. Auch läßt sich die Polizei die Umstände von Konfrontationen nicht mehr von Störern vorgeben, sondern sucht das zeitliche und soziale Vorfeld zu kontrollieren. Die Neuorientierung der Polizei führt nicht zu einem Abbau des staatlichen Gewaltpotentials. Es wird umgestaltet und politisch reflektierter eingesetzt. Die Flexibilität wird möglich, wenn einerseits das Drobpotential erhalten bleibt und andererseits die soziale Kontrolle erweitert wird.

In diese Konzeption fügt sich das polizeiliche Verhalten gegenüber der Friedensbewegung. Diese hatte in der Bevölkerung so viel Sympathie, ihre Aktionen wurden von so vielen Bürgern (»Massen «) getragen, daß die Polizei, hätte sie schärfer darauf reagiert, ihrer sozialen Anerkennung und der Anerkennung der Regierungspolitik geschadet hätte. Auf die Legalität, so wie die herrschende Rechtsprechung sie definiert, hätte die Polizei ein schärferes Vorgehen wohl stützen können; die Legitimität der herrschenden Ordnung hätte sie geschwächt. So ließ sie sich flexibel auf Verhandlungen mit den potentiellen Störern ein und vermied Konfrontationen. Die Gerichtsurteile, die dies zugunsten der Angeklagten berücksichtigen, sind objektiv Folgen der Strategie der Exekutive, die auf Wahrung der politischen Legitimität, der sozialen Anerkennung der Regierung gerichtet ist. Die Abhängigkeit von der Exekutive bedeutet auch: Die Gerichte hätten wohl mehr zu Lasten der Angeklagten entschieden, wenn die polizeiliche Strategie rigider gewesen wäre.

Möglicherweise stützten die gerichtlichen Freisprüche die konfliktvermeidende, integrative Strategie der Polizei auch ideologisch: Verurteilungen hätten vielleicht das polizeiliche Entgegenkommen nachträglich als Täuschung desavouiert. Denkt man diese Spekulation zu Ende, läßt sich in politisch relevanten Fällen annähernd jeder gerichtliche Freispruch als Integrationsunternehmen der Herrschenden verdächtigen. Das mag in einzelnen Situationen angemessen sein. Oft haben aber gerichtliche Erfolge der politischen Opposition auch bestätigende Wirkung für deren Kampf.

Es bleibt der aus den Urteilen selbst zu erschließende Zusammenhang: Sie hängen u. a. vom Verhalten der Exekutive ab. Das ist nicht ungewöhnlich. Über Kriminalisierung entscheiden bekanntlich nicht nur Täter und Richter, sondern auch viele andere Instanzen staatlicher Kontrolle. Man kann aber unterscheiden danach, wieviel Flexibilität, Entscheidungsspielraum, Macht die Gerichtsurteile der Exekutive zugestehen als Vorgabe der gerichtlichen Entscheidung. Aufschlußreich ist insofern ein Urteil des OLG Köln, das prima facie demonstrantenfreundlich orientiert ist ${ }^{6}$. Aus dem Urteil läßt sich auch einiges erschließen über die weiteren Elemente der neuen Polizeistrategie, das Drohpotential und die Vorfeldkontrolle. - Nachdem ein besetztes Haus geräumt worden war, setzten sich Demonstranten davor auf die Straße. Die Polizei hatte den Verkehr aber schon vorher präventiv umgeleitet. Das OLG meint, deshalb seien die Autofahrer von den Demonstranten nicht unmittelbar intensiv, also nach h. M. nicht mit Gewalt, zum Umfahren genötigt worden. Ob die Demonstranten die Fahrer hätten nötigen wollen (Versuch), müsse erst nachgewiesen werden, was das OLG wohl für schwierig hält. Versuch komme »allenfalls in Betracht, wenn der Angeklagte (Demonstrant) keine Kenntnis von der polizeilichen Umleitung hatte und sich vorstellte, durch den Sitzstreik werde konkret auf Kraftfahrer ein unwiderstehlicher Zwang ausgeübt. Im übrigen aber betont das OLG: 
"Eine derartige gewaltsame Sperrung wäre rechtswidrig. Auch das aus Art. 8 GG herzuleitende Demonstrationsrecht gibt nicht das Recht zu einer solchen Verkehrsbehinderung.«

Im Ergebnis sanktioniert das Gericht die Entscheidungsmacht der Polizei. Es hält einerseits daran fest, daß das Blockieren des Verkehrs strafbare Gewaltnötigung sei. Es bestätigt dabei den weiten strafrechtlichen Gewaltbegriff, der sehr viele Verhaltensweisen der Bürger dem Strafrecht unterwirft und damit auch die polizeiliche Eingriffs- und Kontrollkompetenz enorm ausweitet?. Andererseits berücksichtigt das Gericht die Umleitungsaktionen der Polizei als Vermeidung der Nötigung. Es mag sein, daß die Polizei in diesem Fall nicht die Bestrafung der Demonstranten, sondern einen großen Verkehrsstau vermeiden wollte. Gleichwohl zeigt sich, in welcher Weise sie künftig Entscheidungsmacht bezüglich der Kriminalitätsentstehung ausüben kann. Sie muß es nur zu einer kleinen Blockade kommen lassen.

Das OLG hätte der Polizei einen solchen Entscheidungsspielraum nicht zugestehen müssen. Es hätte die Strafbarkeit der Blockade auf verschiedenen rechtlichen Argumentationswegen verneinen können. Einmal hätte es den Begriff der Gewalt in $\$ 240$ StGB enger fassen können, so daß das bloße Blockieren nicht darunter fällt. Damit hätte es sich freilich zur herrschenden Rechtsprechung vor allem des $\mathrm{BGH}^{8}$ in Widerspruch gesetzt. Soweit ersichtlich hat das im Zusammenhang der Demonstrationen der letzten Jahre nur das AG Nürnberg gewagt ${ }^{9}$. Das OLG hätte auch im Hinblick auf Art. 8 GG die Rechtswidrigkeit der Nötigung verneinen können; so haben AG und LG Stuttgart für den Fall entschieden, daß der Verkehr kurzfristig behindert wird ${ }^{10}$. Wenn aber der Gewaltbegriff weit gefaßt und trotz Art. 8 GG die Rechtswidrigkeit bejaht wird, so wird die durch den BGH enorm ausgeweitete staatliche Eingriffskompetenz gegenüber nötigenden Demonstrationen sanktioniert. Es liegt dann u. a. an der Polizei, durch flexibles Verhalten die Kriminalisierung von Fall zu Fall zu vermeiden. Im Ergebnis entsteht ein breites Drohpotential der Exekutive, das zweckrational eingesetzt werden kann zur Steuerung von sozialen Konflikten. - Nun zu den einzelnen Entscheidungen und ihren rechtlichen Kriterien.

\section{Nötigung, Gewalt, Drohung}

Wenn wegen $\ 240$ StGB angeklagt ist, prüfen die Gerichte zunächst, ob die Demonstranten auf der Straße überhaupt eine tatbestandliche Nötigung bewirkt haben. Das ist wie erwähnt fraglich, wenn im jeweiligen Fall die Polizei den Verkehr präventiv umgeleitet hatte. Nach Meinung der Amtsgerichte Erlangen und Stuttgart ${ }^{11}$ wurden die Autofahrer durch die Umleitung zwar genötigt; dieser Erfolg ist den Demonstranten aber nicht zuzurechnen. Er wurde von der Polizei bewirkt. Die entscheidenden Polizeibeamten waren nicht von den Demonstranten beherrscht. Insbesondere wurden sie nicht von den Demonstranten zu Umleitungsmaßnahmen genötigt; sie trafen eine rechtmäßige Ermessensentscheidung, waren also nicht Werkzeuge der Demonstranten, und diese waren nicht »mittelbare Täter $«$ der von den Polizisten bewirkten Nötigung.

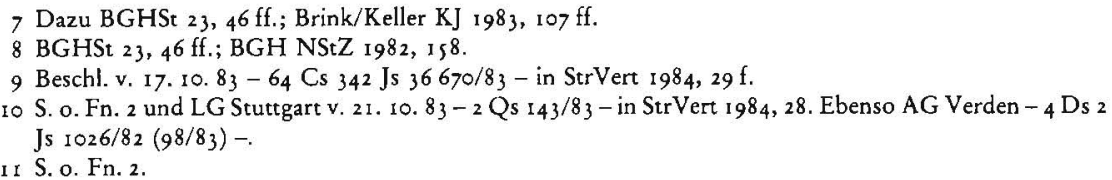


Das OLG Köln ${ }^{12}$ allerdings meint, die Demonstranten seien Täter der in der Umleitung steckenden Nötigung. Jedoch habe diese Nötigung nicht so unmittelbar gewirkt, daß sie als Gewalt bewertet werden müsse. Das OLG geht dabei von dem weiten Gewaltbegriff aus, den der BGH im Laepple-Urteil angenommen hatte. Gewalt fällt danach mit Nötigung, d. h. Freiheitsbeschränkungen, zusammen. Das OLG führt weiter aus:

"Als Ausdruck einer Freiheitsbeschränkung erscheint ein den Nötigungserfolg darstellendes Opferverhalten nur dann, wenn es sunter Druck geschieht (...). Es kommt auf den $\mathrm{Z}_{\text {wangs- }}$ effekt an (...). Einwirkung von Gewalt liegt nur vor, wenn sie von der Person, gegen die sie gerichtet ist, als ein nicht nur seelischer, sondern auch körperlicher $Z_{\text {wang empfunden wird }}$ (...). Stets - auch bei mittelbarer Gewalt - muß eine unmittelbare $Z$ wangseinwirkung auf den Genötigten ausgehen (...). Einer unmittelbaren körperlichen Zwangseinwirkung sind Verkehrsteilnehmer nicht unterworfen, die von der Polizei umgeleitet werden, bevor sie entsprechend dem Willen der Demonstranten durch den Sitzstreik zum Anhalten gezwungen werden. Sie reagieren nur auf eine - zudem rechtmäßige - polizeiliche Verkehrsregelung, die ihre Ursache ebensogut in einem Unfall, in Bauarbeiten oder einer genehmigten Veranstaltung haben kann, möglicherweise sogar in Unkenntnis von dem Sitzstreik der Demonstranten auf der Fahrbahn. Einer unmittelbaren Zwangseinwirkung durch den Sitzstreik werden diese Verkehrsteilnehmer gerade durch die Umleitung entzogen."

Das hier eingeführte Kriterium >körperliche Zwangswirkung könnte den Gewaltbegriff begrenzen. Aber nach Ansicht des OLG wenden auch die sitzenden Demonstranten, die einem Autofahrer die Straße versperren, gegen diesen Gewalt an; anscheinend »empfinden « die Autofahrer den Zwang »körperlich«. Die Bedeutung dieses Wortes wird also sehr weit gefaßt, wie schon in der bisherigen Rechtsprechung ${ }^{13}$. Eine Eingrenzung der Strafbarkeit ist davon nicht zu erwarten.

Weiter soll entscheidend sein die Unmittelbarkeit der Zwangswirkung auf die Autofahrer. Unmittelbarkeit soll zu verstehen sein i. S. einer unmittelbaren Konfrontation zwischen Nötiger und Genötigtem ${ }^{14}$. Warum es darauf ankommen soll, warum also eine Nötigung dann besonders schlimm und strafwürdig wird, wenn der Nötiger dem Genötigten unvermittelt entgegentritt, ist nicht recht verständlich. Das OLG selber stellte zuvor auf die Freiheitsbeschränkung, d. h. die Wirkung beim Genötigten ab. Dies entspricht der BGH-Rechtsprechung ${ }^{15}$. Das Problem der nur mittelbaren Beziehung zwischen Nötiger und Genötigtem in dem vom OLG entschiedenen Fall ist sinnvoller im Horizont der Frage nach mittelbarer Täterschaft zu diskutieren; so die erwähnten Amtsgerichtsentscheidungen.

Der $\mathrm{BGH}^{16}$ äußerte sich zum Gewaltbegriff in einer Revisionsentscheidung betreffend die Blockade des Frankfurter Flughafens. Das OLG Frankfurt ${ }^{16 a}$ hatte A. Schubart, den Initiator der Blockade, wegen versuchter Nötigung der Regierung des Landes Hessen ( $\$$ ros StGB) zu zwei Jahren Freiheitsstrafe verurteilt. Es nahm auch an, Schubart habe schweren Landfriedensbruch begangen ( $\$$ I 25 a StGB), obwohl er bei der Blockade und den sie begleitenden Aktionen nicht anwesend war. Der BGH hob nun die Verurteilung wegen versuchter Nötigung der Landesregierung auf, bestätigte aber, daß Schubart des schweren Landfriedensbruchs schuldig sei; auch

I 2 S. o. Fn. 2.

I3 BGH NStZ 1982, $158=$ NJW 1982, 189 .

I4 Das ist aus den Annahmen des OLG zu erschließen: Wären die Autofahrer nicht umgeleitet worden, so wären sie von den sitzenden Demonstranten, sofern diese nicht gewichen wären, nach Ansicht des OLG unmittelbar (und damit gewaltsam) genötigt worden. Hätten die Polizisten aber selbst bei der präventiven Straßensperrung etwa eine feste Sperreinrichtung verwendet, so wären die Autofahrer von den Demonstranten nach Ansicht des OLG nicht unmittelbar genötigt worden.

15 BGHSt 23, 46 (50, 54).

16 BGHSt $32,165 \mathrm{ff} .=$ NJW $1984,931 \mathrm{ff}$.

16a Urt. v. 19. 1. 83 - I StE 1/82; dazu Brink KJ 1983, $421 \mathrm{ff}$. Dort ist auch der Sachverhalt kurz referiert. 
habe er gegen die behinderten Flughafenbenutzer (einfache) Nötigung ( $\$ 240 \mathrm{StGB}$ ) begangen.

Seine Rechtsprechung zur weiten Fassung des allgemeinen strafrechtlichen Gewaltbegriffs ändert der BGH nicht; er will aber bei Hochverrat und Regierungsnötigung, also bei speziell geregelten politischen Nötigungsdelikten mit besonders hoher Strafdrohung den Gewaltbegriff enger fassen. Andeutungen in diese Richtung finden sich schon in früheren Entscheidungen ${ }^{17}$. Neuerdings hat der BGH auch bei Vergewaltigung, ebenfalls einem speziellen, besonders schweren Nötigungsdelikt, den Gewaltbegriff eingeengt ${ }^{18}$. Dabei zeigt sich auch, daß es nicht immer unproblematisch ist, für die verschiedenen Tatbestände einen je besonderen Gewaltbegriff zu bilden. - Hinsichtlich der Regierungsnötigung stützt der BGH seine Begründung auf gesetzgeberische Erwägungen: ${ }^{19}$

"Die Verfassungsorgane stehen mitten im politischen Leben und sind den Strömungen, Kräften und Gegenkräften, die das politische Geschehen bestimmen, ausgesetzt. Auf sie wirken daher zumindest mittelbar auch die Machtmittel der verschiedensten Art ein, die in den politischen Auseinandersetzungen von den widerstreitenden Gruppen eingesetzt werden." Wenn der Gewaltbegriff bei politischen Delikten so weit gefaßt werde wie im allgemeinen, »würde letztlich die schwierige und im Kern politische Frage, unter welchen Voraussetzungen politische Kampfmaßnahmen verwerflich sind, der Rechtsprechung überbürdet. « ${ }^{\text {I9a }}$

Um dies zu vermeiden, müsse der Gewaltbegriff bei Hochverrat sowie Nötigung von Verfassungsorganen eng gefaßt werden. $\mathrm{Zu}$ fragen ist, warum diese erfreuliche strafrichterliche Selbstbeschränkung nicht auch für den allgemeinen Gewaltbegriff gelten soll. Dessen Anwendung führt dazu, daß praktisch jede Demonstration auf ihre Verwerflichkeit bzw. Sozialwidrigkeit hin überprüft werden kann - eine politische Prüfung. Nach Ansicht des BGH ist Gewalt bei den politischen Nötigungsdelikten nur strafbegründend, wenn sie eine besondere $Z$ wangseignung erreicht:

„Die Zwangswirkung der Gewalt oder der Drohung mit Gewalt entfällt daher, wenn und

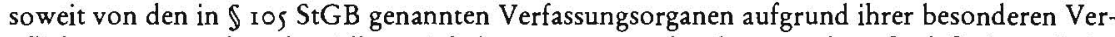
pflichtung gegenüber der Allgemeinheit erwartet werden kann und muß, daß sie auch im Rahmen heftiger politischer Auseinandersetzungen Drucksituationen standhalten. Soll die Regierung eines Landes durch Gewalttätigkeiten gegen Dritte oder Sachen zur Erfüllung bestimmter politischer Forderungen genötigt werden, so sind diese Ausschreitungen somit nur dann Gewalt im Sinne des $₫$ i 25 StGB, wenn der von ihnen ausgehende Druck einen solchen Grad erreicht, daß sich eine verantwortungsbewußte Regierung zur Kapitulation vor der Forderung der Gewalttäter gezwungen sehen kann, um schwerwiegende Schäden für das Gemeinwesen oder einzelne Bürger abzuwenden. « Die von $\mathrm{A}$. Schubart initiierten $\mathrm{Zwangsmaßnahmen}$ erreichten diese Intensität laut BGH noch nicht. "Mit dem mehrstündigen Ausfall eines zentralen Verkehrsflughafens muß auch sonst, z. B. wegen Nebels oder rechtmäßiger Streiks, gerechnet werden. «'sb

Zum allgemeinen Gewaltbegriff bei $₫ 240$ StGB bringt allerdings das AG Nürnberg eine klare Stellungnahme: ${ }^{20}$

„Im Gegensatz zur StA kann das Gericht in der bloßen passiven Resistenz der Blockierer, die es zu keinen sonstigen Unfriedlichkeiten kommen ließen, keine Gewalt erkennen. Die StA folgt hier offensichtlich einem von der herrschenden Rechtsprechung des BGH immer extensiver verstandenen Gewaltbegriff, wonach nicht mehr physische Kraftentfaltung beim Täter (. . .), ja nicht einmal mehr wenigstens physische Zwangswirkung beim Opfer (. . .), sondern schließlich auch jeder nur noch psychisch empfundene $Z$ wang von einigem Gewicht Gewalt sein soll (...).

17 BGHSt 23, $46 \mathrm{ff}$.

I8 BGH NStZ 1981, 390; kritisch dazu Otto JR 1982, I 15; Keller JuS 1984, I1 3 .

19 Der BGH zitiert den Entwurf eines Strafgesetzbuches, 1962 (BT-Ds. IV/650, S. 584 ).

Iga S. o. Fn. 16.

igb S. o. Fn. 16

20 S. o. Fn. 9. 
Der BGH wird mit dieser Gewaltdefinition, die dem - im Schrifttum vereinzelt erklärten (...) - Ziel dient, vermeintliche Strafbarkeitslücken zu schließen (...), aber nicht mehr den Vorstellungen des Rechtsadressaten, des vielzitierten verständigen Durchschnittsbürgers, gerecht, der nach dem allgemeinen Sprachgebrauch mit dem Wort Gewalt die unmittelbare Entfaltung physischer Kraft in aggressivem Handeln gegenüber einem Opfer verbindet (...). Dementsprechend haben auch die Blockierer zu einer 'gewaltfreien Aktion aufgerufen, und die Polizei vermerkte, es sei zu keinen Gewalthandlungen gekommen. Durch diese Abkehr vom sprachlichen Bedeutungsbereich eines Rechtsbegriffs betreibt der BGH darüberhinaus eine bedenkliche - weil durch die Verfassung, Art. 103 Abs. 2 GG, verbotene - Rechtsfortbildung zum Nachteil des Täters, transformiert die strafbare Nötigung durch Verkürzung des Tatbestands auf das Merkmal des Willenszwangs in eein vages Universaldelikt beeinträchtigter Willensbetätigung، (Köhler NJW 1983, I 595 ) aufgrund allgemeinen Strafwürdigkeitsempfindens der Obergerichte (ausgedrückt beispielsweise in sich steigernden unklaren Wendungen wie rnicht billigenswert, unerträgliche Zustände, Terrors im Urt. des OLG Stuttgart [N]W 1969, 1543]).

Trotz des Versuchs des BGH, die offen zugegebene Tatbestandserweiterung durch seine Interpretation von Gewalt in Grenzen zu halten, indem er das ,Kriterium der Intensität der Zwangswirkung ( . . ) schon über den Tatbestand und nicht erst die Rechtswidrigkeit der Nötigung entscheiden läßt, ist dieser Rechtsprechung deshalb aus allgemeinen rechtsstaatlichen Erwägungen prinzipiell nicht zu folgen. (...)*

Derart dezidiert rechtsstaatliche Stellungnahmen zum Gewaltbegriff sind selten. Nach Auskunft von Richtern scheuen sie davor im allgemeinen zurück, weil sie das freisprechende Ergebnis ihrer Entscheidung nicht mit Begründungen stützen wollen, die von Obergerichten voraussichtlich nicht akzeptiert werden. Die Strafbarkeit der Nötigung kann man, wie das AG Stuttgart ${ }^{21}$ zeigt, auch ablehnen, indem der Gewaltbegriff des BGH zwar akzeptiert, aber die Rechtswidrigkeit, d. h. die Verwerflichkeit ( $\$ 240$ Abs. 2 StGB) verneint wird. Die Verwerflichkeit wird auf materiale Gerechtigkeitserwägungen gestützt. Dafür werden die Umstände des konkreten Einzelfalles berücksichtigt und bewertet ${ }^{22}$. Wenn die Verwerflichkeit der Nötigung verneint wird, steckt darin weit weniger beanspruchte Allgemeingültigkeit, als wenn das Gericht den allgemeinen Gewaltbegriff definiert. Wenn es diesen abweichend vom BGH enger faßt, stellt es sich offen und prinzipiell gegen dessen Rechtsprechung. Die Entscheidung zur Verwerflichkeit im Einzelfall kann diesen Konflikt vermeiden.

Im übrigen war mit der engen Fassung des Gewaltbegriffs im Fall des AG Nürnberg die Strafbarkeit wegen Nötigung noch nicht endgültig ausgeschlossen. In Betracht kam noch Nötigung durch Drobung, denn die auf der Straße sitzenden Demonstranten haben den Verkehrsteilnehmern

\#... in Aussicht gestellt, durch Verfolgung ihrer ursprünglichen Absicht, die blockierte Zufahrtsstraße zu benutzen, Menschen überfahren zu müssen, was zweifellos nicht nur für diese selbst, sondern auch für die betreffenden Fahrer ein empfindliches Übel darstellt (Verbot und Verfolgung von Körperverletzung anderer). «2a $^{22 a}$

Das AG nahm also an, $\$ 240$ Abs. I StGB sei durch Drohung erfüllt. Zwingend war dieses Ergebnis nicht. Im Hinblick auf Art. ${ }_{103}$ GG hätte auch der Begriff ,Drohung mit empfindlichem Übel eng gefaßt werden können, etwa ähnlich der bis 1943 geltenden Fassung. Das AG Nürnberg jedoch mußte nun die Verwerflichkeit der Nötigung prüfen.

2 I S. o. Fn. 2.

22 Vgl. Schönke-Schröder-Eser, StGB-Kommentar, 21 . Aufl., 1982, \$240 Rn Is ff., 24a ff.

22a S. o. Fn. 2. 
Die gründlichste Stellungnahme zu diesem Problem hat das AG Stuttgart gegeben. Der Begriff >Verwerflichkeit « wird durch das Grundrecht der Demonstrationsfreiheit näher bestimmt. Das AG betont, daß Verkehrsbehinderungen normale Folge von Demonstrationen sind, also nicht per se strafbar sein können. Wichtig ist der Hinweis, daß Demonstrationen unfriedlich erst sind, wenn sie saufrührerisch oder gewalttätig، verlaufen; demnach werden die Unfriedlichkeit und die Grenze des Grundrechts nicht durch den Gewaltbegriff des Strafrechts bestimmt, wie der BGH es im Laepple-Urteil vorgezeichnet hatte. Vielmehr ist das Strafrecht durch das Grundrecht zu bestimmen. Die Entscheidung des AG muß hier nicht genauer referiert werden. Sie ist in KJ 1983 , H. 3 , S. $340 \mathrm{ff}$. abgedruckt. Sie verneint die Rechtswidrigkeit. Das LG Stuttgart ${ }^{23}$ ist ihr gefolgt, das AG Verden ${ }^{24}$ hat ebenso entschieden. Der $\mathrm{BGH}^{25}$ läßt neuerdings in der Entscheidung zur Frankfurter Flughafenblockade zur Frage der Verwerflichkeit immerhin offen, »ob Demonstrationen, die um der größeren Öffentlichkeitswirkung wegen darauf angelegt sind, die Bewegungs- und Handlungsfreiheit anderer durch Gewalt zu beeinträchtigen, stets oder nur unter zusätzlichen Voraussetzungen nach $\$ 240$ StGB strafbar sind." Solche zusätzlichen Voraussetzungen sind nach Ansicht des BGH gegeben, wenn die Demonstranten Gewalttätigkeiten i. S. des $\$$ I25 StGB begehen; die Demonstranten am Frankfurter Flughafen hatten »Barrikaden errichtet, diese gegen die zur Räumung eingesetzten Ordnungskräfte verteidigt und Polizeibeamten, die die Zugänge freihalten wollten, aktiven Widerstand geleistet. "Das sind nach Ansicht des BGH Gewalttätigkeiten.

Dokumentiert werden soll noch die Stellungnahme des AG Nürnberg ${ }^{26}$, weil sie das Verhältnis zur früheren BGH-Rechtsprechung deutlich macht.

"Weder das Fehlen allgemeiner Rechtfertigungsgründe für die hier durchgeführte Demonstration (...) noch die bloße Verletzung von Spielregeln der Demokratie führen jedoch schon zur erhöhten sittlichen Mißbilligung der Aktion. Der BGH hat mehrfach (so auch in BGHSt 17 , 388) erklärt, der Richter habe auf das Rechtsempfinden des Volkes zu achten, der Begriff der Verwerflichkeit in $\$ 240$ Abs. 2 StGB knüpfe an sozialethische Vorstellungen an, weshalb das rechtlich Verwerfliche nicht einseitig in dem angewandten Mittel oder in dem angestrebten Zweck, sondern in der Beziehung beider zueinander zu suchen sei. Wenn der BGH unter dieser Prämisse die Rechtswidrigkeit einer körperlichen Attacke eines Freiers auf eine Dirne verneint, durch die er I0,- DM zurückerhalten wollte, auf die er glaubte Anspruch zu haben, so wäre es mit einem solchen Urt. unvereinbar, den Versuch ernstzunehmender und um das immaterielle Wohl ihrer Mitmenschen besorgter Mitbürger, jedenfalls nicht von der Hand zu weisende drohende Gefahren effektiv ins allgemeine Bewußtsein zu bringen, als verwerflich zu brandmarken, wenn die Belästigung anderer so vergleichsweise minimal bleibt wie hier der Fall. (...)

Den hier Demonstrierenden ging es nicht um die Nichterhöhung von Straßenbahntarifen (wie in BGHSt 23, 46 ff.) oder die Dekonzentration im Pressewesen (wie in OLG Stuttgart NJW 1969, 1543) sondern um das Leben der Mitbürger, das vor dem reibungslosen Funktionieren des Straßenverkehrs rangieren sollte. Der deutliche Hinweis auf vitale Bedrohungen durch Rüstungswettlauf und Atomwaffen ist, jedenfalls solange dabei nicht annähernd gleiche Werte verletzt werden, wie die, für welche demonstriert wird, weder von smilitanten Minderheiten geübter Terror، (wie BGHSt 23, 57) noch sittlich verwerflich.«

In dieser Entscheidung ist zutreffend hervorgehoben, daß auch bei Überschreitung der Demonstrationsfreiheit die Verwerflichkeit i. S. des $\$ 240$ Abs. 2 StGB noch 
nicht ohne weiteres gegeben ist. Denn diese Vorschrift setzt einen über die allgemeine Rechtswidrigkeit hinausgehenden erhöhten, materialen Unwert der Nötigung voraus ${ }^{27}$. Darauf geht z. B. das OLG Köln ${ }^{28}$ nicht ein, wenn es nur nach einer Rechtfertigung durch Art. 8 GG fragt und sie apodiktisch ablehnt. Zu bemerken bleibt, daß die Entscheidungen vom AG und LG Stuttgart sowie vom AG Verden nur kurze Verkehrsbehinderungen ( 12 Minuten) betrafen; das AG Nürnberg hatte es zwar mit einer längeren Blockade zu tun; es wurden jedoch nur wenige Bürger behindert. Nach den Begründungen ist nicht ganz sicher, ob die Gerichte ebenso entschieden hätten bei gravierenderen Behinderungen. Das OLG Köln hält ohne nähere Begründung eine Blockade von $I^{1 / 2}$ Stunden für rechtswidrig.

\section{Hintermänner des Landfriedensbruchs}

Bei der Blockade des Frankfurter Flughafens beging A. Schubart nach Ansicht des BGH auch schweren Landfriedensbruch in mittelbarer Täterschaft. Die Demonstranten, die er zur Blockade aufgerufen hatte, errichteten Barrikaden und leisteten der Polizei aktiven Widerstand, begingen also Landfriedensbruch. Dabei war Schubart zwar nicht anwesend, er hatte aber nach Ansicht des OLG und des BGH die Übergriffe vorausgesehen und, weil er bei den Demonstranten maßgebliche Autorität hatte, beherrscht. - Nach der bis 1970 geltenden weiten Fassung des $\$$ I 2 S StGB haftete nur, wer in der gewalttätigen Menge anwesend war. Die Neufassung hat, wie bisher angenommen wurde, den $\$ I 25 eingeschränkt. Der BGH dagegen meint, weil in die Neufassung der Terminus sals Täter< eingefügt worden sei, werde auf $\$ 25$ Abs. I StGB verwiesen; danach haften auch vom Tatort abwesende mittelbare Täter. Zur Begründung führt der BGH weiter aus:

„Da Strafgrund nicht mehr der Anschluß an eine unfriedliche Menge, sondern die Beteiligung an den Gewalttätigkeiten oder Bedrohungen ist, besteht nach der Neufassung kein Anlaß mehr, den ortsabwesenden Befehlsgeber, Organisator oder geistigen Anführer von der Strafbarkeit auszunehmen, wenn und soweit die aus der Menge verübten Gewalttätigkeiten oder Bedrohungen seinem Tatwillen entsprechen und unter seiner Tatherrschaft begangen werden, ihm also nach allgemeinen Grundsätzen als eigene Tat zuzurechnen sind. Insoweit dehnt die Neufassung daher die Strafbarkeit gegenüber dem alten Tatbestand aus. Diese Auslegung hat auch der Regierungsvertreter bei den Beratungen des neuen $\$ I2s StGB für richtig gehalten. $\aleph^{28 a}$

Diese Argumentation läßt sich bezweifeln. Der Terminus ,Täter ‘ hat in $\$$ I 25 andere Bedeutung als in $\$ 2$ s. Das wird von der h. M. auch anerkannt: Täter i. S. des $\$ 2$ s StGB sind im Kontext des $\$$ I2s StGB Täter und Teilnehmer. Der Täterbegriff des $\$ 125$ kann also abweichend vom $\$ 25$ gefaßt werden, wenn es dem »Strafgrund « des Tatbestandes entspricht ${ }^{29}$. Insofern verweist der BGH auf das Begehen von Gewalttätigkeiten; dafür könnten in der Tat auch mittelbare Täter haften. Aber $\$$ I 25 pönalisiert offensichtlich nicht das Begehen von Gewalttätigkeiten per se, sondern nur für den Fall, daß sie aus einer Menge begangen werden. Nach der Konzeption des Gesetzes ist diese Situation, die Eigendynamik der Menge, besonders gefährlich und

27 Der BGH (23, 46 ff.; NStZ 1982, 158 f.) meint allerdings, bei Gewaltanwendung sei die Rechtswidrigkeit der Nötigung "praktisch « indiziert. Praktisch durchhalten läßt sich dies jedoch kaum, wenn der weite Gewaltbegriff zugrunde gelegt wird; vgl. u. a. F. C. Schroeder JuS 1982, $49 \mathrm{Iff}$.

28 S. o. Fn. 2.

28a S. o. Fn. 16.

29 Fincke, Das Verhältnis des Allgemeinen zum Besonderen Teil des Strafrechts, Berlin 1975, S. 65 ff.; Lenckner in Schönke-Schröder(s. o. Fn. 22) $\$ 125$ Rn 16 ff.; Blei JA 1970, 616; Rudolphi in Syst. Komm. $\$ 125 \operatorname{Rn} 13$. 
begründet mit das Sonderdelikt des $\$$ I 25. Gewalttätigkeit und Anwesenheit in der

Menge sind also zusammen die Strafgründe des \$ 25 . Deshalb kann der Abwesende nicht der Täter sein ${ }^{30}$. Dem mögen einige Äußerungen der Mitarbeiter der Gesetzgebung entgegenstehen. Bei anderen Auslegungsfragen kümmert sich die Rechtsprechung um solche Äußerungen wenig. Bemerkenswert ist aber, daß der BGH auf die Argumente, die seiner Auslegung entgegenstehen und in der Literatur längst verbreitet sind, mit keinem Wort eingeht. Daß nach der BGH-Entscheidung auch "Hintermänner" des Landfriedensbruchs erfaßt werden, kann gefährlich werden u. a. für Leute, die bei Demonstrationen Gehör finden und sie fördern. Denn wenn es später aus der Menge zu Gewalttätigkeiten kommt, ist u. U. schwer auszuschließen, sie hätten dies vorausgesehen und beherrscht.

Karl Leb

30 Blei, Lenckner, Rudolphi zit. in Fn. 29.

Soeben erschienen:

Wolfgang Däubler (Hrsg.)

\section{Arbeitskampfrecht}

Das Handbuch zum Thema Streik, Aussperrung, Warnstreik, „Neue Beweglichkeit“, Solidaritätsstreik, Boykott, Betriebsbesetzung u. a. mit der neuesten Rechtsprechung.

1984, 634 S., 48,- DM

In jeder Buchhandlung oder

Tel. 072 21 / 3441 - Telex 781201

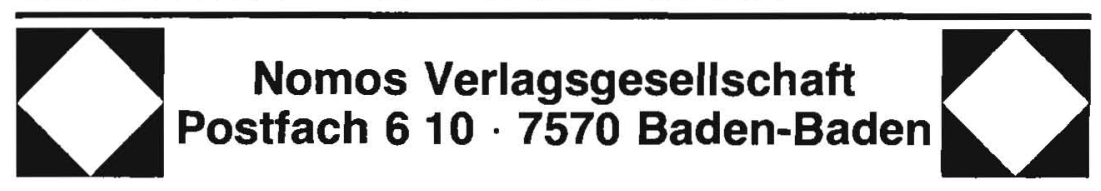

